\title{
EL DILEMA DE LA REPRESENTATIVIDAD POLÍTICA
}

\section{THE DILEMMA OF POLITICAL REPRESENTATIVENESS}

\author{
nariño@esap.edu.co \\ (92) 7224318- 7224376- 7224551
}

\section{RESUMEN}

Para muchos estudiosos de la ciencia política moderna (Schmitt (1928), Pitkin (1985), Manin (1999)), la representación política es un concepto que define la política contemporánea y la forma como ésta se desarrolla. Cada uno de ellos desde perspectivas jurídicas y filosóficas, entienden que la representación política al incluir aspectos como los de individuo libre, racional y autónomo, el derecho al voto y la participación del proceso decisional por parte del congreso, componen al estado moderno.

Esta investigación se enmarcó en reconocer la situación socialmente problemática de la representatividad política teniendo en cuenta que, en la actualidad, los partidos políticos en particular, son objeto de desconfianza e incredulidad y la fragmentación combinada con un aumento del personalismo del sistema de partidos en Colombia (Ungar y Arturo, 2004). Las inconformidades ante el funcionamiento de los sistemas de partidos y de las instituciones en donde hacen presencia, han suscitado un debate alrededor de la crisis de representación que los afecta, viéndose permeados por la permanencia de los partidos tradicionales en el poder o el surgimiento de nuevos movimientos sociales de índole étnico, política, sindicalista, de origen popular.

Identificando que en Colombia existe una compleja red de incentivos clientelistas, además del creciente abstencionismo y el bajo compromiso de los militantes, influidos por las prebendas de caudillos y varones electorales. Concluyendo, que el reto de los partidos alternativos y tradicionales, hoy en día, es construir niveles de confianza y cultura politica con el fin de ganar y aportar 
por la verdadera representatividad política

Palabras clave: representación política, partidos políticos, carismáticos, programático clienteslistas.

\section{ABSTRACT}

(Schmitt (1928), Pitkin (1985), Manin (1999)), political representation is a concept that defines contemporary politics and the way in which it develops. Each of them with juridical and philosophical perspectives, understand that political representation feeds free, rational and autonomous individuals, the right to vote and the participation of the decision process by the part of the congress, make up the modern state.

This research was framed in recognizing the socially problematic situation of the political representation taking into account that, at present, the political parties in particular, the object of distrust and disbelief and the fragmentation combined with the increase of the personalism of the party system in Colombia (Ungar and Arturo, 2004). Nonconformities with the functioning of party systems and the institutions where they are present have sparked a debate around the crisis of the representation that affects them, seeing them begged for the permanence of traditional parties in power or Emergence of new social movements of an ethnic, political, syndicalist, popular origin.

Identifying that in Colombia there is a network of clientelistic incentives, in addition to the growing abstentionism and low commitment of the militants, influenced by the prebendas of caudillos and electoral men. Concluding, that the challenge of alternative and traditional parties, today, is to build levels of trust and political culture in order to win and contribute for the true political representation

Keywords: political representation, political parties, charismatics, clientelist, programs 


\section{INTRODUCCIÓN}

Se reconoce que el concepto de representación, como tantos otros conceptos fundamentales de la política, es «esencialmente controvertido», se considera que es posible extraer de todas sus interpretaciones un mínimo común que se identifica con «actuar en beneficio de los representados». La ventaja de presentar una definición de este tipo es que permitiría realizar análisis empíricos de lo que realmente hacen los representantes y emitir juicios sobre la calidad de la representación con independencia de lo se considera que deba ser la relación representante/representado. Por lo anterior muchos autores parte de una concepción extremadamente simple de lo que significa la representación, y tanto la reflexión teórica como el estudio de los comportamientos políticos, definiendo que los representantes pueden actuar legítimamente de forma distinta.

En tal sentido y queriendo hacer un acercamiento a este concepto, se toman los aportes de Pitkin (1985) y Mainwarning (2006), quienes indican que la representación política es la actuación sustantiva por otros, es decir, la relación del principal agente por el cual A (el ciudadano elector representado) autoriza a $\mathrm{B}$ (el electo o representante) a actuar en su interés. Aunque es común observar la codependencia de A y B alrededor del interés colectivo, en la práctica la representación que ejerce $B$ resulta más compleja de lo que se cree. El representante permanentemente se enfrenta a un dilema a la hora de decidir sobre qué intereses representar, si los del electorado o los institucionales.

Como se ve el concepto de representación y más aún su ejercicio tiene especial controversia tanto en la significación lingüística como en su ejercicio dinámico en las actuales democracias participativas. En este contexto y teniendo en cuenta el interés que tiene tratar el tema de representatividad política en los territorios y principalmente en zonas de frontera como los es el departamento de Nariño, se quizo hacer un estudio en tres municipio a saber Ipiales, Ricaurte y Tumaco para identificar las principales características en el ejercicio de la representación política de los diferentes cuerpos colegiados.

\section{DESARROLLO DE LA PONENCIA}

Hay que mencionar en primera instancia, que: La teoría del sistema 
político redefine la función que tenía la división de poderes en las teorías políticas tradicionales; poderes que representaban en el campo político diferenciaciones clasistas propias del ordenamiento social, pasan a convertirse en funciones o estructuras de un mismo ordenamiento sistémico. Esta diferenciación funcional entre representación (legislativo), administración (ejecutivo) y control (judicial), refleja una particular forma de conducción de politicidad en el proceso decisional, por medio de la cual la influencia política de las demandas sociales y económicas ingresan por el sistema de representaciones en el cual sufren una dinámica de procesamiento y de abstracción política; los intereses restringidos o parciales (partidarios) se articulan y priorizan en un programa de realizaciones que consiste en una orientación de acciones para la administración pública, la misma que deberá precisarlas ulteriormente a través de procedimientos técnicos específicos.

Mientras en el campo representativo es legítima la presencia de los intereses sociales articulan una propia "racionalidad parlamentaria", en el campo de la administración pública dicho influjo se reduce hasta desaparecer en el acatamiento y en la conformidad a las normas de los procedimientos administrativos.

En esta caracterización, el influjo político en la función judicial está terminantemente prohibido; de esta manera se consume el punto más alto de abstracción política, al permitir que esta función se convierta en legítima instancia de control constitucional, logrando de esta forma un adecuado equilibrio organizacional interno para el sistema político. En base al logro de los requisitos antes anotados, el sistema político logra diferenciarse del ámbito de lo social, autonomizarse respecto de las presiones sociales y económicas, y puede finalmente absolver su tarea central, que es la de producir decisiones políticas.

De esta manera se cumplen las funciones de interacción concretas entre el sistema político y los actores sociales a nivel más general, en cambio, podemos hablar de que el sistema político, al procesar demandas y devolverlas al ambiente social bajo forma de decisiones, lo que hace es "producir poder social", esto es, desarrollar, cultivar y generar bases de poder, incrementando la capacidad de reproducción social al fomentar en los actores sociales comportamientos que vehiculizan y promueven la combinación entre elaboración de demandas y 
decisiones políticas. (Echeverria, 1997, p. 83)

Por otra parte la representación política es un concepto que define la política contemporánea y la forma como ésta se desarrolla. Bajo esta premisa, ha sido estudiada por autores como Hobbes (1651), Mill (1861), Madison (1788), Schmitt (1928), Pitkin (1985) y Manin (1999) por mencionar algunos ejemplos. Cada uno de ellos desde perspectivas jurídicas y filosóficas, entiende que la representación política al incluir aspectos como los de individuo libre, racional y autónomo, el derecho al voto -el cual permite la interacción entre el representante y el representado y la participación del proceso decisional por parte del Congreso, componen al Estado moderno.

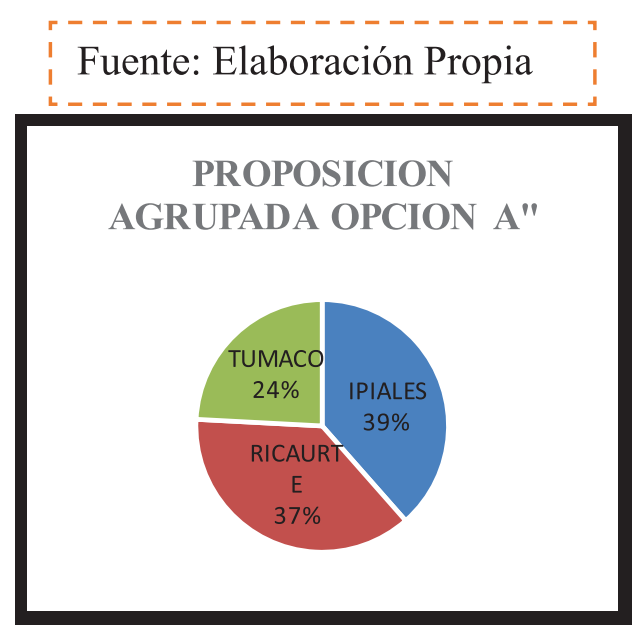

Buscando hacer un acercamiento al concepto, se toman los aportes de Pitkin (1985) y Mainwaring (2006), quienes señalan que la representación política es la actuación sustantiva por otros, es decir, la relación del principalagente por el cual A (el principal) autoriza a B (el agente) a actuar en su interés. Aunque es común observar la codependencia de A y B alrededor del interés colectivo, en la práctica, la representación que ejerce B resulta más compleja de lo que se cree. El representante permanentemente se enfrenta a un dilema a la hora de decidir sobre qué intereses representar, si los del electorado o los institucionales. Esta divergencia muestra al representante tanto como un presentador especial de causas, quien tiene en cuenta las preferencias subjetivas y necesidades de sus electores; como un agente racional, motivado de acuerdo a 
una serie de incentivos al interior del sistema político. En otras palabras, actuar o hablar en nombre de otro (acción social) constituye una decisión compuesta por intereses, valores, medios y fines que funcionan dentro de una estructura racionalizada e institucionalizada.

En consecuencia, el legislador electo no representa a sus votantes en cualquier asunto, ni tampoco lo hace por sí mismo aisladamente. Por el contrario, el representante moderno actúa dentro de una elaborada red de presiones, demandas y obligaciones, respondiendo a cálculos y compromisos respecto a diversos actores. Por consiguiente, el representante es considerado un presentador especial de causas, un agente de su localidad que debe tener en cuenta las consideraciones subjetivas de sus electores y las consideraciones racionales del sistema político en el que se encuentra inserto. Es por ello que sólo a partir de los procesos institucionalizados y engranados en las pautas de conductas culturales cómo la representación adquiere una forma real (políticas públicas, gestión, gobernabilidad, gestión, etc.) (Pitkin, 1967).

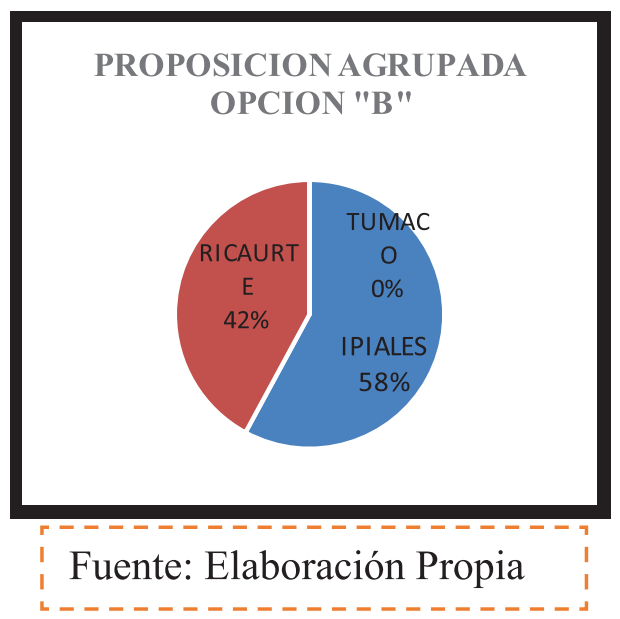

Así las cosas, la relación de los concejales con el votante no es una relación unidireccional. Ésta aborda a múltiples intermediarios, como son las organizaciones sociales y comunitarias, entre estas están: las Juntas de Acción Comunal, las Juntas Administradoras Locales, los consejos consultivos de comuna y corregimiento, los consejos territoriales de planeación, los gremios, organizaciones religiosas, organizaciones juveniles, organizaciones de mujeres 64 
y los movimientos culturales, son con quienes se establecen diferentes tipos de vínculos o formas de representación política, las cuales tienen como objetivo la lucha por la distribución de los recursos y el poder en lo sub nacional. Estos vínculos conectan a los ciudadanos con sus representantes, indicando las formas en que operan las instituciones democráticas en lo sub nacional con respecto a cómo resuelven la tensión permanente entre el bien común y el interés institucional.

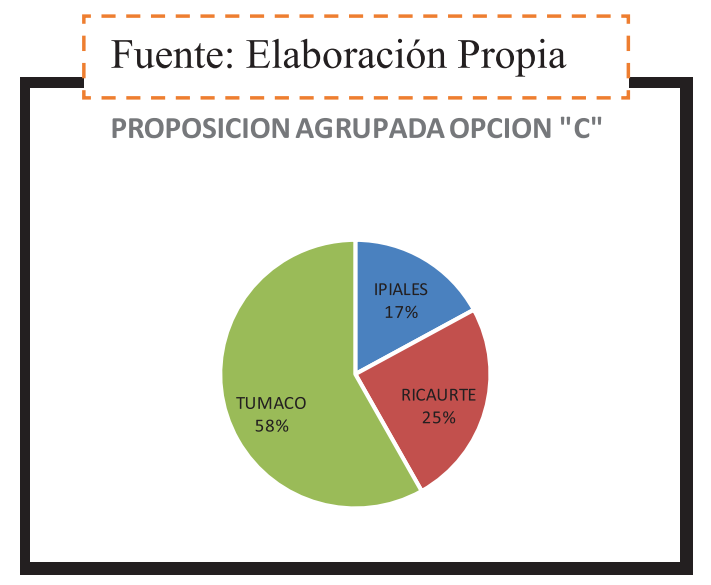

Kitschelt (2000) hace una clasificación en la que propone tres tipos de vínculos y sus respectivos procedimientos para resolver el dilema de la representación. Una primera de orden programático, la cual se distingue por cultivar principios ideológicos y técnico- organizativos al interior de los partidos, contando con una agenda política unificada y la suficiente capacidad para generar cohesión interna entre sus integrantes. En este contexto, la relación entre el principal y el agente -en palabras de Mainwaring (2006)-, opera con base a la formulación de paquetes legislativos, decretos y mandatos de política pública que priorizan acciones en el largo plazo, sobre un mapa completo de necesidades de los ciudadanos.

Tal y como se pudo constatar en la investigación "Análisis Comparado de la representatividad política en zona de frontera Colombo Ecuatoriana periodo comprendido desde 1990-2015” para los municipios de Tumaco, Ipiales y Ricaurte en Colombia, donde se obtuvieron los siguientes resultados 
con respecto a la pregunta ¿Qué es la representación política?. Lo cual da como opción la proposición agrupada respecto al orden programático y como en observa en el grafico inmediatamente anterior, el municipio de Ipiales responde que "Es la participación mediante la cual un grupo de personas elige un líder consecuente con los principios y fines del partido en coherencia con la agenda política para que busque el bienestar y desarrollo de las comunidades”. En el municipio de Ricaurte "se representa por principios ideológicos en coherencia con un programa de gobierno del cual se deriva las alianzas estratégicas", En Tumaco el concejo respondió así "Una representación política debe servir de una base programática y estructurada para así poder generar progreso en las comunidades, es el buen manejo de los recursos", en promedio un $47,72 \%$ elige un actuar programático.

Un segundo tipo de vínculo es el clientelar, basado en una estructura de intercambio de favores e incentivos otorgados de manera asimétrica antes y después de las elecciones. La entrega de beneficios es cada vez más especializada, por cuanto se pasa de un clientelismo tradicional compuesto por una red de lealtades entre el cliente con el patrón, a un clientelismo moderno, de carácter anónimo y capaz de poner a competir a los actores por los incentivos, es así como para la investigación antes mencionada se obtuvieron los siguientes resultados teniendo como opción "B” la clientelar y como se puede observar en la gráfica, Ipiales afirma que "Se presenta cuando los candidatos a corporaciones públicas buscan ganar las elecciones usando mecanismos como la compra y venta de votos perdiendo los principios del partido", en Ricaurte determinan que "Es por intercambio de favores", En Tumaco se presenta un hecho bastante significativo, ¡no se presente una relación Clientelistaj, lo que representa el promedio del $13,63 \%$.

Finalmente, cuando las relaciones son carismáticas-personalistas, el líder establece un vínculo con la ciudadanía respaldado por la habilidad para persuadir al electorado mediante sus propias cualidades. A causa de esto, el representante no se encarga de invertir en una estructura organizativa porque básicamente no lo requiere, le basta con una estrategia de promesas con el potencial para resolver problemas en el corto plazo. Para la investigación en mención se obtuvieron los siguientes resultados para la opción "C" para el orden clientelar, como se puede determinar en la gráfica, los concejales de Ipiales un $18,75 \%$ responden que "Los representantes a través de sus habilidades 
de persuasión convencen al electorado y luego no retribuyen a la sociedad", en Ricaurte establece un $27,27 \%$ que "no se vota por el partido sino por el candidato el considera que representa a la comunidad", En Tumaco consigna un $64 \%$ que "la participación política debe ser libre pero adecuada donde el objetivo general debe ser el bienestar de la comunidad".

Pues bien, utilizando esta tipología presentada por Kitschelt (2000), el dilema de la representación política se resuelve según los incentivos empleados, los mismos que cumplen con delimitar la capacidad de representación al interior del sistema. En síntesis, no es lo mismo resolver el dilema bajo las condiciones de un personalismo extremo, o hacerlo en el marco de una democracia partidista donde las decisiones tomadas son el resultado de la acción colectiva.

Para ilustrar el tema de la representación política a partir del caso colombiano, la reforma constitucional de 1991 introdujo cambios importantes en el sistema político, logrando, primero, incrementar la participación electoral de las minorías étnicas, regionales y políticas; segundo, disminuir el clientelismo con raíces en el Frente Nacional; y alterar la naturaleza de la representación con efectos en el ejercicio del poder al nivel nacional y regional. De acuerdo a esto, se produjo una apertura en el sistema de participación, apareciendo nuevos sectores en la arena política nacional, quienes a su vez, demostraron el compromiso de formular iniciativas programáticas al interior del Senado y las circunscripciones regionales. Por tanto, la Constitución Política de 1991 se convierte en un hito histórico que tuvo efectos significativos en el número de partidos (Sartori, 1987), en las corrientes de interacción del sistema, las tácticas de competencia y la oposición de partidos (Hoyos, 2005).

Sin embargo, el dilema de la representación en el cual los partidos políticos son los puentes entre el Estado y el electorado, no se resuelve de acuerdo a vínculos programáticos, por el contrario, a pesar del proceso de democratización del sistema, en Colombia han existido dos escenarios. Un primero con la prevalencia de los vínculos clientelares y la personalización de la política, tanto por parte de los partidos tradicionales, como de las nuevas fuerzas. Esto causó dos fenómenos que se alimentan el uno al otro. Por un lado, una personalización extrema de la vida política, y por otro, una representación política cada vez más particularista, orientada a sectores más específicos de la población y dando lugar a una concentración de votantes proporcional a 
los incentivos otorgados con el fin de obtener apoyo en las urnas (Pizarro Leongómez, 2008). Y un segundo, basado en cómo la violencia (grupos armados) permeó las esferas políticas con la llegada del paramilitarismo (parapolítica) y dineros del narcotráfico para la financiación de campañas electorales.

En conclusión, para Colombia las diferentes tendencias políticas, el tipo de régimen político, el sistema de partidos y su comportamiento en el nivel nacional, es posible afirmar que al interior de su sistema político conviven mecanismos tanto programáticos, corporativos, regionales, armados, clientelares, personalistas, étnicos, religiosos (Pizarro, 2010) y las combinaciones derivadas entre ellos, sirven para caracterizar el contexto y las condiciones políticas, económicas e institucionales.

Otro aspecto de gran importancia para el desarrollo de la representatividad política es el Índice de Percepción sobre la Gobernabilidad propuesta por Álvarez la cual indica la existencia de correlación entre la percepción ciudadana y la calificación del pobre desempeño de las administraciones locales. Cabe anotar que la estructura del Índice de Percepción sobre la Gobernabilidad democrática (IPG) se organizó con base en cinco dimensiones interdependientes: 1) la opinión ciudadana respecto al régimen político, con lo cual se estableció una asociación clara entre la baja percepción de gobernabilidad y desconfianza por parte de la ciudadanía, 2) la cultura y la participación política, 3) la confianza en las instituciones, relacionado con el pobre desempeño de las administraciones locales, los cuerpos colegiados y entidades de control 4) la valoración de la concentración del poder y 5) las percepciones sobre la situación socioeconómica.

Es así como y después de indagar a diferentes cuerpos colegiados respecto a la pregunta ¿Qué es un Sistema Político? La cual tiene relación directa con gobernabilidad, ya que cuando se habla de régimen, sistema político o forma de gobierno lo conveniente es hacer una precisión conceptual puesto que los concejales utilizan indiscriminadamente los conceptos que son sinónimos dentro de la ciencia política, es importante que exista un elemento determinante cual es la estructura de poder por cuanto se refiere a la participación y a la representación de la ciudadanía como se puede ver en el siguiente tabla la distribución porcentual en las respuestas que se presentan 68 
en forma de proposiciones agrupadas en el Municipio de Ipiales el 62,5\% ,opta por opción "b", que hace referencia "A la organización del estado basado en la relación entre instituciones organizaciones y ciudadanía que busca el buen funcionamiento de la administración pública (basada en leyes, obligaciones y derechos)".

De igual manera en el municipio de Ricaurte el 63,63\% de los concejales afirma en su proposición agrupada "es una organización que le da estructura a la participación ciudadana y a la buena relación con la comunidad”, Así mismo el cuerpo colegiado en el Municipio de Tumaco el 70,6\% afirman que "el sistema político es el medio donde se dirige la comunidad en general es el hilo conductor para incluir a todas las partes que lo conforman para el logro de los objetivos en cada uno de ellos", En desacuerdo con la opción anterior y que optaron por la opción "c" en las proposiciones agrupadas para el concejo de Ipiales el $25 \%$ argumenta que es "Conjunto de actores que participativamente buscan el seguimiento y control para dar solución a los problemas de la comunidad", a lo cual se suman a la opción "c" en el municipio de Ricaurte 9,1\% los cuales afirman que la inclusión "inclusión de todos los sectores que eligen un representantes", los concejales de Tumaco adhieren a esta respuesta el $29,4 \%$ bajo el siguiente criterio "es un componente de organización para la buena marcha del desarrollo del país, es el conjunto de Instituciones grupos y procesos políticos que interactúan en un entorno", en promedio $22,72 \%$ se pude calificar que tienen un insuficiente conocimiento, para Ipiales el 12,5\% opta por la opción "a” que afirman que "Es la unión de los grupos políticos donde se materializan sus ideologías mediante la participación en lo político y lo social", en el municipio de Ricaurte el 27,27\% "es la mejor herramienta para darle poder Político a las personas", en el municipio de Tumaco ninguno de los concejales optan por esta opción y finalmente el 11,33\% su respuesta es totalmente incorrecta. 
Tabla 1: Opinión sistema político concejales municipales Colombia

\begin{tabular}{|c|c|c|c|}
\hline \multirow{2}{*}{$\begin{array}{l}\text { PREGUNTA: } 1 \\
\text { ¿QUE ES UN } \\
\text { SISTEMA } \\
\text { POLITICO? }\end{array}$} & A & B & $\mathrm{C}$ \\
\hline & $\begin{array}{l}\text { Es la designación de } \\
\text { poder Político que } \\
\text { representan en el } \\
\text { campo político } \\
\text { diferenciaciones } \\
\text { clasistas propias del } \\
\text { ordenamiento social }\end{array}$ & $\begin{array}{l}\text { Es el conjunto de } \\
\text { relaciones que se } \\
\text { establecen entre } \\
\text { instituciones, } \\
\text { organizaciones y } \\
\text { ciudadanía, y a partir } \\
\text { de las cuales se } \\
\text { estructura una forma } \\
\text { peculiar de dirección, } \\
\text { gestión y } \\
\text { competencias } \\
\text { políticas. }\end{array}$ & $\begin{array}{l}\text { Es un conjunto de } \\
\text { elementos: la } \\
\text { institucionalidad, } \\
\text { grupos de presión, la } \\
\text { iglesia, los gremios y } \\
\text { la población que } \\
\text { exige derechos } \\
\text { sociales y políticos }\end{array}$ \\
\hline \multirow{2}{*}{$\begin{array}{l}\text { PROPOSICIONES } \\
\text { AGRUPADAS } \\
\text { PREGUNTA } \\
\text { IPIALES }\end{array}$} & $A(2) 12,5 \%$ & $62,5 \%$ & C (4) \\
\hline & $\begin{array}{l}\text { Es la unión de los } \\
\text { grupos políticos done } \\
\text { se materializan sus }\end{array}$ & $\begin{array}{l}\text { Es la organización } \\
\text { del estado basado en } \\
\text { la relación entre }\end{array}$ & $\begin{array}{l}\text { Conjunto de actores } \\
\text { que } \\
\text { participativamente }\end{array}$ \\
\hline
\end{tabular}




\begin{tabular}{|c|c|c|c|}
\hline & $\begin{array}{l}\text { ideologías mediante } \\
\text { la participación en lo } \\
\text { político y lo social. }\end{array}$ & $\begin{array}{l}\text { instituciones } \\
\text { organizaciones y } \\
\text { ciudadanía que busca } \\
\text { el buen } \\
\text { funcionamiento de la } \\
\text { administración } \\
\text { pública (basada en } \\
\text { leyes, obligaciones y } \\
\text { derechos). }\end{array}$ & $\begin{array}{l}\text { buscan el seguimiento } \\
\text { y control para dar } \\
\text { solución a los } \\
\text { problemas de la } \\
\text { comunidad. }\end{array}$ \\
\hline \multirow{2}{*}{$\begin{array}{l}\text { PROPOSICIONES } \\
\text { AGRUPADAS } \\
\text { PREGUNTA } \\
\text { RICAURTE }\end{array}$} & $\begin{array}{l}\text { A (3) } \\
\mathbf{2 7 , 2 7 \%}\end{array}$ & $63,63 \%$ & $\begin{array}{ll} & \text { C (1) } \\
9,1 \% & \end{array}$ \\
\hline & $\begin{array}{l}\text { Es la mejor } \\
\text { herramienta para } \\
\text { darle poder Político a } \\
\text { las personas }\end{array}$ & $\begin{array}{l}\text { Es una organización } \\
\text { que le da estructura a } \\
\text { la participación } \\
\text { ciudadana y a la } \\
\text { buena relación con la } \\
\text { comunidad }\end{array}$ & $\begin{array}{l}\text { Inclusión de todos los } \\
\text { sectores que eligen un } \\
\text { representantes }\end{array}$ \\
\hline \multirow{2}{*}{$\begin{array}{l}\text { PROPOSICIONES } \\
\text { AGRUPADAS } \\
\text { PREGUNTA } \\
\text { TUMACO }\end{array}$} & $\begin{array}{ll} & \text { A } \\
& \end{array}$ & $\begin{array}{ll} & \mathrm{B}(12) \\
\mathbf{7 0 , 6} \% & \end{array}$ & $29,4 \%$ \\
\hline & & $\begin{array}{l}\text { El sistema político es } \\
\text { el medio donde se } \\
\text { dirige la comunidad } \\
\text { en general es el hilo } \\
\text { conductor para } \\
\text { incluir a todas las } \\
\text { partes que lo } \\
\text { conforman para el } \\
\text { logro de los } \\
\text { objetivos en cada } \\
\text { uno de ellos }\end{array}$ & $\begin{array}{l}\text { Es un componente de } \\
\text { organización para la } \\
\text { buena marcha del } \\
\text { desarrollo del país, es } \\
\text { el conjunto de } \\
\text { Instituciones grupos } \\
\text { y procesos políticos } \\
\text { que interactúan en un } \\
\text { entorno }\end{array}$ \\
\hline
\end{tabular}


Según lo investigado para remediar esta situación y teniendo en cuenta que con el proceso de transición a la democracia, los gobiernos locales tienen, ante sí, los retos de lograr eficacia en sus políticas públicas que les garanticen la confianza de la sociedad civil. La participación de los ciudadanos en la gestión municipal y estatal es el factor más importante para el desarrollo de la democracia. Ésta se nutre cada vez que los ciudadanos toman parte de los cambios políticos, económicos, sociales y culturales. Po todo lo anterior la gobernabilidad democrática en el futuro dependerá de: la construcción de un sistema que invite a la cooperación, la construcción de nuevas instituciones políticas que provoquen incentivos colectivo y tengan mayor injerencia en la toma de decisiones y la adecuación del marco legal en torno al desarrollo de las capacidades ciudadanas, en la misma medida que las institucionales.

\section{CONCLUSIONES Y RECOMENDACIONES.}

De acuerdo con la interpretación de Alejandro Monsivais, cuando hace la propuesta de la configuración política representativa interpretando los postulados de Hanna Pitkin, lo cual es acorde a lo encontrado en esta investigación ya que menciona que los planteamientos de representatividad deben ser adaptados a las realidades concretas y en diferentes sentidos y parte de las diversas investigaciones que se tiene en la teoría política sobre la representación política. Al encontrarse con la disyuntiva acerca que si "actuar en lugar de los representados" significa obedecer un mandato expreso o se interroga también si el representante debe actuar con plena independencia (representatividad desde lo programático), los autores asumen una posición clara haciendo explicito que representar no significa hacerse cargo de algo en un sentido paternalista (representatividad desde lo clientelista), ni tampoco representa " quien solo consulta y sigue indicaciones de tipo personal (representatividad desde lo carismático), la representación política tiene lugar cuando se actúa conforme al interés de los representados de una manera responsables hacia ellos.

\section{BIBLIOGRAFÍA.}

KITSCHELT, Herbert. (2000). Linkages between Citizens and Politicians in Democratic Polities, en Comparative Political Studies, Vol. 33 No. 6/7. pp. 845-879.

FREIDENBERG, Flavia (2013). Los cambios del sistema de partidos ecuatoriano en treinta años de democracia (1978-2010). Salamanca: Ediciones 
Universidad de Salamanca. Disponible en: http://works.bepress.com/ flavia_freidenberg/73/

MAINWARING, Scott, Ana María Bejarano, y Eduardo Pizarro Leongómez. (2008). La crisis de la representación democrática en los países andinos: un panorama general, en La crisis de la representación democrática en los países andinos, Bogotá: Grupo Editorial Norma. pp. 23-86. 